\title{
A NOTE ON THE ESTABLISHMENT AND MAINTENANCE OF THE NICHOLS STRAIN OF VIRULENT T. PALLIDUM IN RABBITS IN WARM COUNTRIES*
}

\author{
BY \\ C. W. CHACKO, L. YOGISWARI, AND K. N. GOPALAN \\ V.D. Department, Madras Medical College
}

The Nichols strain of virulent $T$. pallidum, isolated and maintained by serial transmission in live rabbit testes, is being used as the source of specific antigen in the Treponemal Immobilization (TPI) and Treponemal Agglutination (TPA) tests for syphilis. It has been stressed by investigators in this field that the rabbits, after inoculation with the treponemata, should be kept in air-conditioned $\left(18\right.$ to $20^{\circ} \mathrm{C}$.) rooms to produce lesions within a comparatively short incubation period and thereby make available suitable specific antigen. Treponemes apparently prefer a cool environment and cool tissues to multiply and thrive well. It has been possible on actual trial, however, to produce infection by the intratesticular route in rabbits kept in the natural warm $\left(24.6\right.$ to $33.4^{\circ} \mathrm{C}$.) animal room conditions at Madras, India, within an incubation period of about 10 days comparable with that obtained in a cool or airconditioned environment. The Nichols strain has been maintained successfully by serial transmission in rabbit testes, at regular intervals of about 10 days, throughout the last 12 months.

\section{Material and Methods}

The Nichols strain of $T$. pallidum was obtained by air mail from the State Serum Institute, Copenhagen. It arrived in Madras about 72 hrs after dispatch as frozen rabbit testes syphilomata, kept frozen in thickwalled, large, glass test-tubes, packed in solid carbondioxide in a thermos vacuum jar.

Immediately on arrival, the testes were rapidly thawed in running cold water, and cut into thin slices with a pair of sharp scissors. A pestle and mortar was used to grind them into an emulsion with a little fine sand and about $5 \mathrm{ml}$. per testis of 25 per cent. normal rabbit serum in saline. The emulsion was then lightly centrifuged at 1,500 revolutions per minute for $7 \mathrm{~min}$. to sediment the gross particles of tissue and sand. A drop of supernatant fluid showed thirty to forty actively motile treponemes per field of the dark-field microscope, with the high-dry objective, corresponding to 30 to $\mathbf{4 0}$ million treponemes

* Received for publication July 19, 1955. per $\mathrm{ml}$. of the suspension. With a tuberculin syringe and a 24-gauge 1-in. needle, $0.75 \mathrm{ml}$. of the suspension was inoculated in equal halves through each of the two poles of each testis of young adult rabbits of mixed breed. The rabbits were kept in individual cages in the animal room (not air-conditioned), and were observed daily by palpation of the testes for signs of infection. By the eighth to ninth day, diffusely indurated syphilomata of the testes were found, and specific infection was confirmed by dark-field examination of testicular fluid drawn by syringe and needle puncture for live treponemes. Within $24 \mathrm{hrs}$ the rabbits were killed by air embolism into the ear vein. The scrotum was clamped and cut off. The testes were enucleated, sliced, and ground into an emulsion as before, with $5 \mathrm{ml}$. of 25 per cent. normal rabbit serum in saline per testis, so that the centrifuged suspension contained thirty to forty treponemes per dark field. This was passaged intrate iticularly, as before, into the next series of rabbits. All these procedures were carried out as quickly as possible under aseptic conditions, with surgical gloves.

\section{Results}

The Nichols strain of $T$. pallidum was received during February, 1954, and by February, 1955, 122 rabbits in 35 series (average three rabbits) were used. During this period the average mean minimum and maximum temperatures noted in Madras were 24.7 and $33.4^{\circ} \mathrm{C}$. respectively. The mean incubation period of infection, as calculated by finding the mean of averages for every passage, was 10.76 days (standard deviation $\pm 2 \cdot 27$ ). At the height of summer in Madras, namely, during April, May, and June, the average mean minimum and maximum temperatures were $27 \cdot 8$. and $37^{\circ} \mathrm{C}$. respectively, when the corresponding average mean incubation period was 11.05 days (standard deviation \pm 2.25 ). The difference in the incubation periods is not considered significant $(t=0.2 ; P=0.563)$. Several batches of a stable specifically agglutinable antigen suspension were prepared from these treponemes for treponemal agglutination tests for syphilis. 


\section{Discussion}

Rich, Chesney, and Turner (1933) and Cumberland and Turner (1949), working with experimental rabbit syphilis, suggested that the production of a clinical lesion in syphilis is the end-result of a logarithmic multiplication of treponemes in the inoculum. The treponemes have little resistance to high temperatures, the rate of multiplication is slower, and more time is required for the accumulation of sufficient organisms to produce a clinical lesion in syphilis in a warm environment. Hollander and Turner (1954) in their experiments with the incubation period and early development of syphilitic lesions in rabbits, found that environmental temperature had an important influence on the course of experimental treponemal infection, and that this influence was exerted through its effect on the local temperature of tissues. This was particularly noticed when rabbits were infected intradermally on the back. The dark-field positive primary lesions invariably appeared within significantly longer incubation periods when the rabbits were maintained in natural warm conditions of 29 to $35^{\circ} \mathrm{C}$. as compared with rabbits kept in natural cool conditions in the winter months and/or in air-conditioned rooms at 18 to $20^{\circ} \mathrm{C}$. However, in one similar experiment, no significant difference was noted between the incubation period of infections producing testicular syphilomata in a warm environment and that in a cool environment. Moore and Quick (1924) found that the temperature of the mammalian testis is normally maintained at a level considerably below the abdominal temperature, the muscles of the scrotum apparently controlling it by the elevation or lowering of the testes as required. Obviously, the testes in rabbits may be able to maintain a relatively constant optimum temperature for normal multiplication of treponemes in warm and cool surroundings. These findings seem to have been confirmed by us by the successful intratesticular infection of rabbits with an average incubation period of about 10 days, and maintenance by serial passage during 12 months at intervals of about 10 days. The incubation period of infection, the type of syphilitic orchitis produced, and the number of treponemes, suitable for preparing specific antigen, obtained from the testes of infected Madras rabbits, compare well with those seen by the senior author (C. W. C.) in the U.S.A. in rabbits kept in air-conditioned rooms, and also with those obtained by him in his similar experiments under natural cool conditions in London. However, it is stressed that in these circumstances the number of treponemes in the infective inoculum is very important and that a sufficient density of at least 30 to 40 million per $\mathrm{ml}$. must be inoculated into the testes to obtain satisfactory results.

\section{Conclusion}

It is concluded that, to isolate and maintain the virulent strain of $T$. pallidum for preparing suitable specific antigen for tests for syphilis, and for studying experimental syphilis and the biology of the virulent treponemes, it may not be necessary to maintain rabbits in air-conditioned animal rooms in tropical warm countries provided they are inoculated intratesticularly with an adequately infective inoculum.

\section{REFERENCES}

Cumberland, M. C., and Turner, T. B. (1949). Amer. J. Syph., 33, 201. Hollander, D. H., and Turner, T. B. (1954). Ibid., 38, 489.

Moore, C. R., and Quick, W. J. (1924). Amer. J. Physiol., 68, 70 Rich, A. R., Chesney, A. M., and Turner, T. B. (1933). Bull. Johns Hopk. Hosp., 52, 179. 